\title{
Recombinant Clostridium difficile Toxin Fragments as Carrier Protein for PSII Surface Polysaccharide Preserve Their Neutralizing Activity
}

Maria R. Romano ${ }^{1}$, Rosanna Leuzzi ${ }^{1}$, Emilia Cappelletti ${ }^{2}$, Marta Tontini ${ }^{1}$, Alberto Nilo ${ }^{1}$, Daniela Proietti ${ }^{1}$, Francesco Berti ${ }^{1}$, Paolo Costantino ${ }^{1}$, Roberto Adamo ${ }^{1}$ and Maria Scarselli ${ }^{1, *}$

1 Novartis Vaccines, Via Fiorentina 1, Siena 53100, Italy;

E-Mails: maria_rosaria.romano@novartis.com (M.R.R.); rosanna.leuzzi@novartis.com (R.L.); marta.tontini@novartis.com (M.T.); alberto.nilo@novartis.com (A.N.); daniela.proietti@novartis.com (D.P.); francesco.berti@novartis.com (F.B.); paolo.costantino@novartis.com (P.C.); roberto.adamo@novartis.com (R.A.)

2 Novartis Vaccines Institute for Global Health, Via Fiorentina 1, Siena 53100, Italy; E-Mail: emilia.cappelletti@novartis.com

* Author to whom correspondence should be addressed; E-Mail: maria.scarselli@novartis.com; Tel.: +39-577-53-9467.

Received: 6 February 2014; in revised form: 6 March 2014 / Accepted: 9 April 2014 / Published: 22 April 2014

Abstract: Clostridium difficile is a Gram-positive bacterium and is the most commonly diagnosed cause of hospital-associated and antimicrobial-associated diarrhea. Despite the emergence of epidemic $C$. difficile strains having led to an increase in the incidence of the disease, a vaccine against this pathogen is not currently available. $C$. difficile strains produce two main toxins (TcdA and TcdB) and express three highly complex cell-surface polysaccharides (PSI, PSII and PSIII). PSII is the more abundantly expressed by most C. difficile ribotypes offering the opportunity of the development of a carbohydrate-based vaccine. In this paper, we evaluate the efficacy, in naive mice model, of PSII glycoconjugates where recombinant toxins A and B fragments (TcdA_B2 and TcdB_GT respectively) have been used as carriers. Both glycoconjugates elicited IgG titers anti-PSII although only the TcdB_GT conjugate induced a response comparable to that obtained with $\mathrm{CRM}_{197}$. Moreover, TcdA_B2 and TcdB_GT conjugated to PSII retained the ability to elicit IgG with neutralizing activity against the respective toxins. These results are a crucial proof of concept for the development of glycoconjugate vaccines against $C$. difficile infection (CDI) 
that combine different $C$. difficile antigens to potentially prevent bacterial colonization of the gut and neutralize toxin activity.

Keywords: Clostridium difficile; glycoconjugate; PSII polysaccharide; recombinant toxin fragment

\section{Introduction}

Clostridium difficile is a Gram-positive, spore-forming and toxin-producing anaerobic gastrointestinal pathogen that is the major cause of antibiotic-associated colitis. C. difficile has been isolated from several domestic and nondomestic animal species, and has been associated with diarrhea in horses, pigs, dogs and cats. In humans, $C$. difficile associated diarrhea (CDAD) is the most commonly diagnosed cause of hospital-associated and antimicrobial-associated diarrhea [1].

C. difficile infection (CDI) has grown tremendously since 1978, and over the last decade, the incidence and severity of CDI has increased significantly and affected new patient groups. Today, the disease represents a major social and economic burden [2]. Since 2005, CDI has been increasingly reported among young, healthy individuals residing in the community. An estimated $20 \%$ to $28 \%$ of CDI is community associated with an incidence of 20 to 50 cases per 100,000 population in the United States, Sweden and England [3].

At the moment, there is no vaccine against $C$. difficile, despite the increase in the incidence of the disease observed in the last decades [4,5].

The virulence of $C$. difficile is conferred primarily by two large exotoxins, toxins $\mathrm{A}$ and $\mathrm{B}$, and there is evidence that protection against severe CDI is mediated by systemic antibodies to TcdA and TcdB [6-8]. Both toxins present three distinct functional domains: an N-terminal enzymatic domain consisting of glucosyl-transferase (GT) and cysteine protease (CP) moieties, a central translocation (T) domain that mediates import into host cells and a C-terminal receptor binding domain (RBD) with 38 tandem repeats [9].

Although a number of studies have demonstrated that anti-toxin circulating antibodies are effective in the treatment of severe CDI [10,11], supporting the key role of toxin immunity in preventing the lethal outcome of this infection, the use of toxoid-based vaccines in humans has been limited for a long time. Recently, preparations of formaldehyde-inactivated toxoid from $C$. difficile culture supernatants have been able to confer protective immunity in clinical trials [11-14].

To overcome the safety issues potentially associated to the large-scale production of toxoids, such as exposure to toxins and spores, the use of recombinant proteins has been proposed as an attractive alternative for development of vaccines against CDAD [15]. Several studies have demonstrated the ability of recombinant toxin fragments to induce robust immunity against lethal challenge with $C$. difficile. In particular, TcdA and TcdB RBDs, cloned and purified from a variety of hosts, have been proven to induce both systemic and mucosal neutralizing antibodies in animal models [16-18]. Our group has recently shown that co-administration of a cell binding domain fragment of TcdA (TcdA_B1) and the glucosyltransferase moiety of TcdB (TcdB_GT) can induce systemic IgGs, neutralizing both the respective toxins and protecting vaccinated animals from death in hamster animal model of lethal infection. The presence of anti-TcdA and TcdB antibodies was assessed in gut contents, suggesting that systemic 
vaccination with this pair of recombinant polypeptides can limit the disease caused by toxin production during CDI [19]. However, anti-toxins antibodies elicited by toxin fragments are not able to limit the level of bacterial load in the gut [20].

Recently, it has been shown that $C$. difficile vegetative cells express three highly complex polysaccharides on their cell surface, named PSI, PSII and PSIII. Among those three carbohydrates, PSII has been found to be the more abundantly expressed by the hypervirulent rybotype O27 [21]. The PSII is a polysaccharide composed of a hexaglycosyl phosphate repeating unit [-6)- $\beta$-D-Glcp-(13)- $\beta$-D-GalpNAc-(1-4)- $\alpha$-D-Glcp-(1-4)-[ $\beta$-D-Glcp-(1-3)]- $\beta$-D-GalpNAc-(1-3)- $\alpha$-D-Manp-(1-P] [22].

We have previously employed the high-resolution magic angle spinning (HR-MAS) NMR on vegetative whole cells from a collection of clinical isolates and have detected PSII on the surface of different rybotypes, such as $001,018,027,078$ and 126 [23]. The list of isolates analyzed by this technique has been further updated, detecting PSII in a number of clinical and environmental isolates, including strain 630 [24]. Therefore, PSII is as a surface antigen conserved among the most common strains and can represent a relevant target for the development of a carbohydrate-based vaccine.

In confocal microscopy, examination of vegetative cells using anti-PSII antibodies revealed that PSII does not appear as a typical thick and even bacterial capsule; then it can be hypothesized that the polysaccharide is present either as cell wall-linked polysaccharide not bound to peptidoglycan or as a conjugate with lipoteichoic acids [21,24].

Interestingly, strain 630 and the hypervirulent strain R20291 can form in vitro structured biofilms, where the presence of PSII could be detected by antibodies against the phosphorylated hexaglycosyl repeating unit [25]. This suggests that extracellular PSII could play a role in determining the biofilm's architecture of $C$. difficile as component of extracellular matrix.

Glycans are $\mathrm{T}$ cell independent antigens, but they can be turned into molecules able to evoke a $\mathrm{T}$ cell memory response following conjugation to a carrier protein [26]. Anti unconjugated PSII IgM antibodies have been generated in pregnant pigs vaccinated with a non-adjuvanted PSII containing an average of six repeating units [27]. PSII, after conjugation to $\mathrm{CRM}_{197}$ (non-toxic mutant of diphtheria toxin) [28], a carrier protein widely used for the manufacturing of glycoconjugate vaccines [29], was formulated with the adjuvant MF59 and tested in Balb/C mice, inducing high levels of specific anti carbohydrate $\mathrm{IgG}$, a class of antibodies which is generally relevant to induce protection against the sugar coated pathogens [23]. Therefore, conjugation of PSII to the carrier protein could ensure, as expected, the IgM-to-IgG switch. Noteworthy, glycoarray analysis has demonstrated that specific IgA antibodies in the stool of patients infected with $C$. difficile can recognize the nonphosphorylated PSII hexasaccharide hapten, suggesting that under exposure to PSII the human immune system may furnish a mucosal response against carbohydrate epitopes from PSII [30].

The co-administration of multiple $C$. difficile antigens, by using recombinant toxin fragments conjugated to PSII could have the potential to prevent colonization and protect against $C$. difficile disease. We envisaged in conjugation of PSII to toxins as a strategy to ensure co-delivery of the two antigens, using the toxin as carrier protein for the polysaccharide. With this aim, we have evaluated the immunological response of PSII-toxin based glycoconjugates in mouse, investigating the possible double role of the two TcdA_B2 and TcdB_GT fragments, as carrier protein for the PSII polysaccharide and antigens able to elicit antibodies with toxin neutralizing activity. 


\section{Results and Discussion}

\subsection{PSII-Toxins Conjugates}

The two C. difficile recombinant toxin fragments, TcdA_B2 and TcdB_GT, derive from a fragment design of TcdA and TcdB assisted by computer modeling. The tripartite organization of both toxins, the GT and CP regions were sub-cloned, and the receptor RBDs were sub-divided into several fragments. On the basis of previous immunological results, we selected the two TcdA_B2 and TcdB_GT fragments as carrier protein for the PSII polysaccharide [19].

PSII is composed of hexaglycosyl repeating units hold together by phosphodiester bonds [22], and the assigned structure has been confirmed by synthesis of the non-reducing end terminal phosphorylated repeating unit [31].

Pure PSII with an average degree of polymerization (avDP) of 15, obtained from fermentation of the R20291 strain (Stoke Mandeville -ribotype 027) as previously reported [23], was conjugated to the two $C$. difficile recombinant fragments derived from TcdA and TcdB after chemical modification of the mannose sugar of the repeating unit at the reducing end. PSII was first reduced with $\mathrm{NaBH}_{4}$ and then oxidized with sodium periodate to introduce an aldehyde group useful for the coupling to the lysine residues of the protein by reductive amination [23] (Figure 1).

Figure 1. Conjugation scheme of PSII-toxins.
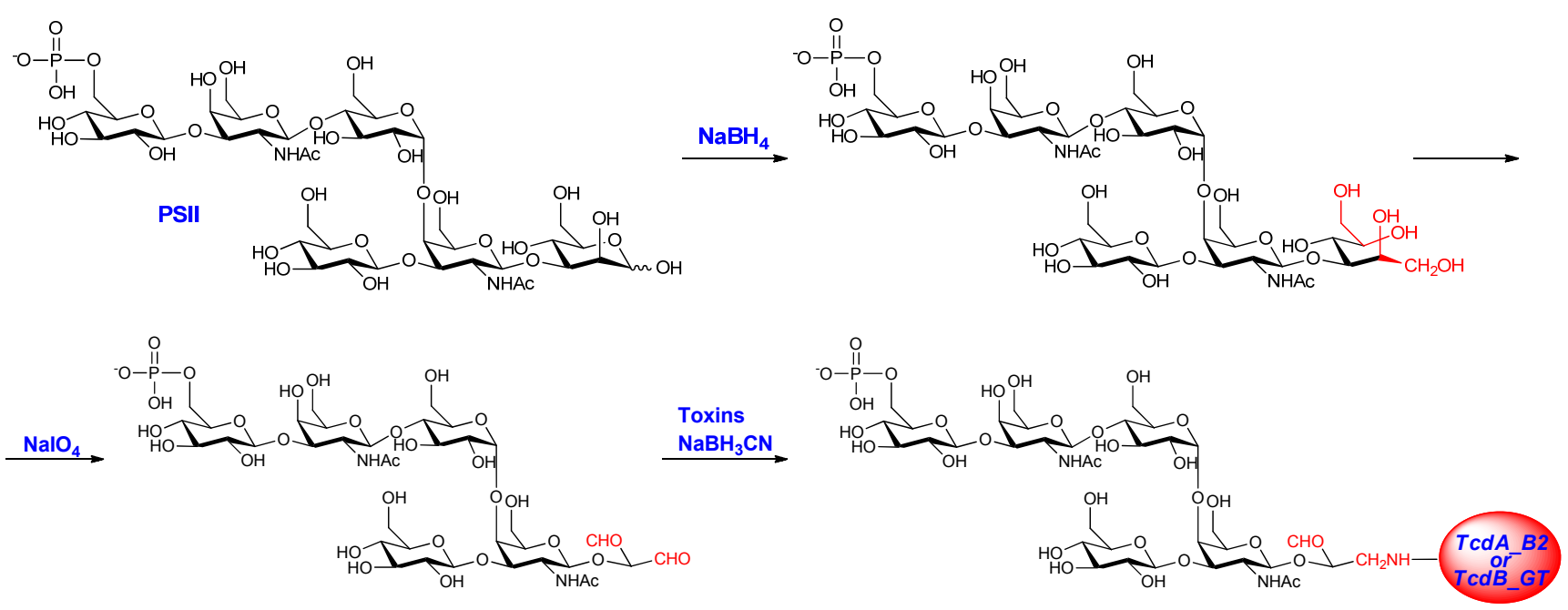

The occurrence of complete conjugation was assessed by sodium dodecyl sulfate-polyacrylamide gel electrophoresis (SDS-PAGE), and confirmed by the formation of a broad smear and the concomitant disappearance of the narrow band of the proteins (Figure 2). Subsequently, the glycoconjugates were purified by size exclusion chromatography to remove unbound saccharide and analyzed for their protein content and in terms of total and free saccharide by HPAEC-PAD HPLC as described in literature [23].

Table 1 summarizes the physico-chemical characterization of the purified glycoconjugates in term of total and free saccharide and protein content, compared to the PSII-CRM 197 conjugate of which the preparation and the characterization were previously reported [23]. Notably, the degrees of glycosylation, ranging from 0.2 to $0.3(\mathrm{w} / \mathrm{w})$, were comparable for both the products. 
Figure 2. Sodium dodecyl sulfate-polyacrylamide gel electrophoresis (SDS-PAGE) of PSII-toxin conjugates 1: TcdA_B2 fragment protein; 2: PSII-TcdA_B2 conjugate; 3: TcdB_GT fragment protein; and 4: PSII-TcdB_GT conjugate.

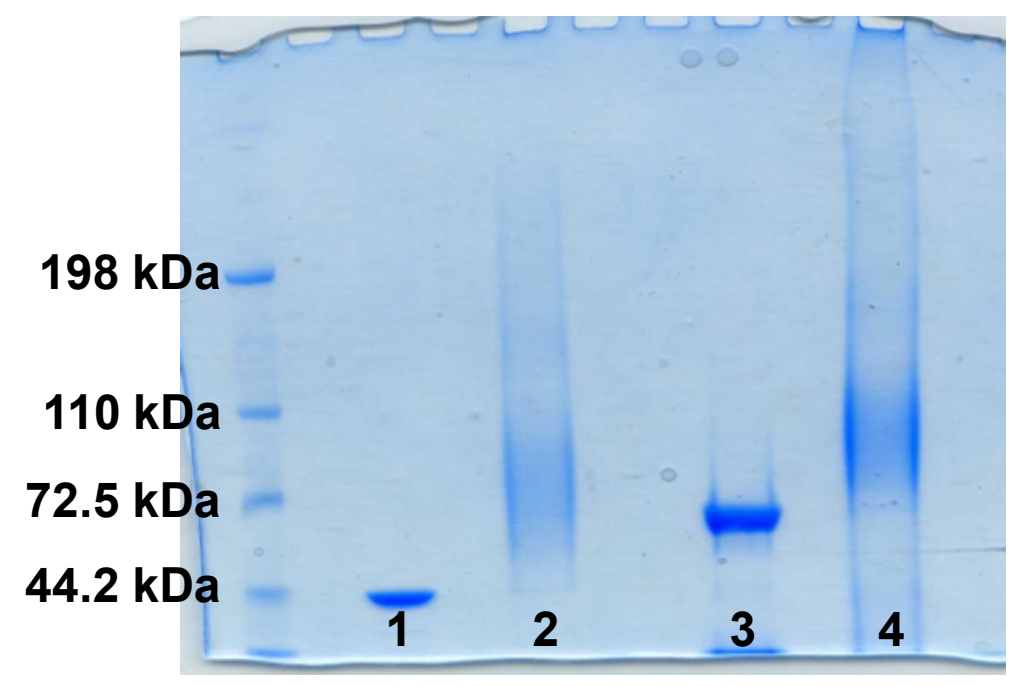

Table 1. Characteristics of PSII conjugates. avDP: average degree of polymerization.

\begin{tabular}{cccc}
\hline Conjugates & PSII avDP & Free saccharide (\%) & Saccharide/protein (w/w) \\
\hline PSII-TcdA_B2 & 15 & 7.7 & 0.28 \\
PSII-TcdB_GT & 15 & 22.7 & 0.33 \\
PSII-CRM ${ }_{197}^{*}$ & 21 & 11.2 & 0.24 \\
\hline
\end{tabular}

Note: * Characterization previously described [23].

\subsection{Immunological Evaluation of PSII-Toxins Conjugates}

To assess the ability of conjugates to induce anti-PSII antibodies, groups of eight female BALB/c mice were intraperitoneally immunized three times with $2.5 \mu \mathrm{g}$ carbohydrate based doses of conjugates, at three week-interval between the first and the second dose and two week-interval from the second and the third dose. The conjugates were formulated with the adjuvant MF59, an oil in water emulsion frequently used for seasonal flu vaccination [32]. Adjuvant alone in phosphate buffered saline (PBS) was used as a negative control, while the PSII-CRM ${ }_{197}$ conjugate previously shown capable of inducing a robust anti-polysaccharide immune response [23], and the TcdA_B2 and TcdB_GT fragment proteins already shown to be highly immunogenic [19], were used as a positive control.

Sera obtained after two weeks from the third dose (post 3 sera) were analyzed by enzyme-linked immunosorbent assay (ELISA) for their content of anti-PSII and anti-Toxin IgGs. Additionally, the functionality of the anti-Toxin antibodies was investigated in vitro to assess the capacity to neutralize the cytotoxicity of TcdA and TcdB.

The PSII-TcdB_GT conjugate was highly immunogenic, eliciting anti-PSII IgG titres comparable to those obtained with the PSII-CRM 197 conjugate. Conversely, PSII-TcdA_B2 conjugate induced an anti-polysaccharide response significantly lower than the $\mathrm{CRM}_{197}$ conjugate ( $\mathrm{p}$ 0.006), where $\operatorname{IgGs}$ against PSII were induced in three mice only (Figure 3). These results evidenced a better capability of the TcdB_GT fragment protein to function as carrier for the PSII moiety in comparison to the TcdA_B2 peptide. 
Figure 3. Anti-PSII IgG levels detected in individual post 3 sera of BALB/c mice; each dot represents single mouse sera; vertical bars indicate geometric mean titers of each group with 95\% statistical confidence intervals as red bars. ELISA: enzyme-linked immunosorbent assay.

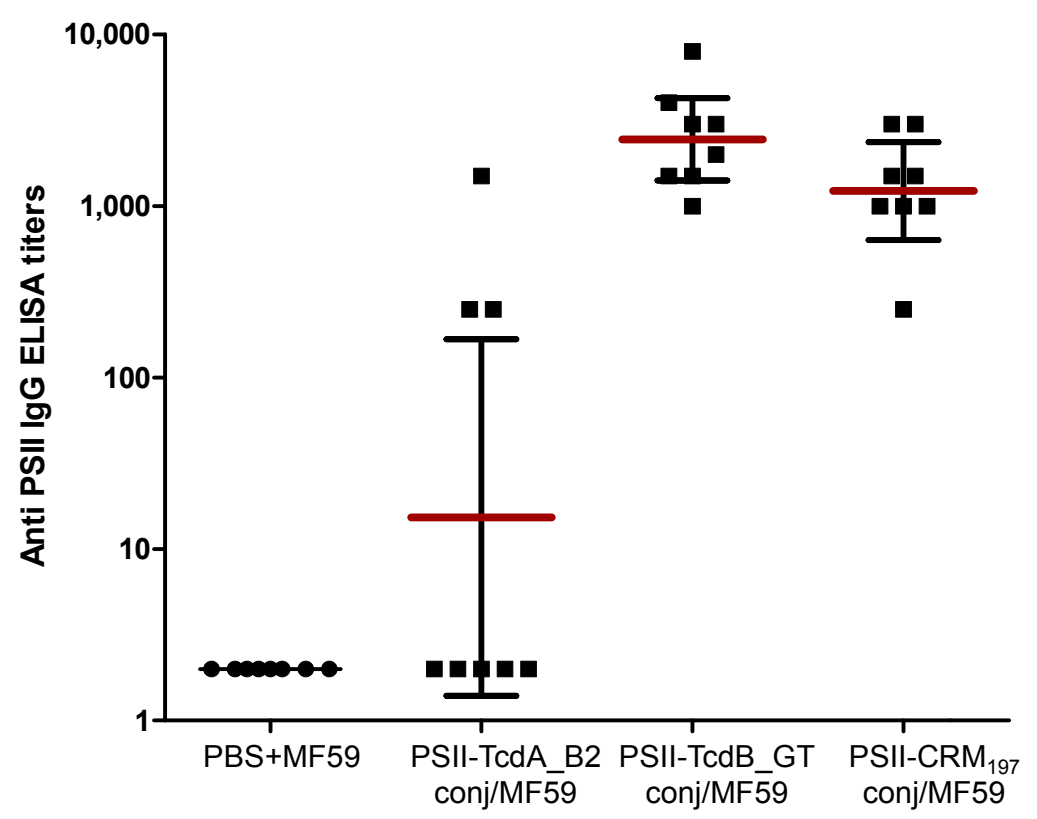

The antibodies response to the TcdA_B2 and TcdB_GT fragment proteins as carrier was measured and compared with the IgG titers obtained with unconjugated TcdA_B2 and TcdB_GT fragment proteins.

As shown in Table 2, both conjugated toxin fragments evoked a robust anti protein response. However, unconjugated TcdA_B2 elicited significantly higher anti toxin fragment antibodies in comparison to the corresponding PSII conjugate ( $p$ 0.002), while TcdB_GT induced IgGs levels comparable to the conjugated form.

Table 2. IgG levels detected in individual post 3 sera of BALB/c mice against TcdA_B2 and TcdB_GT coating. PBS: phosphate buffered saline.

\begin{tabular}{ccc}
\hline Antigen & IgG titer (geometric mean titer) & 95\% confidence interval \\
\hline PBS & 2 & - \\
TcdA_B2 fragment protein & $1,024,000$ & $374,868-4,023,000$ \\
PSII-TcdA_B2 conjugate & 51,499 & $28,721-109,279$ \\
TcdB_GT fragment protein & 206,317 & $47,610-400,390$ \\
PSII-TcdB_GT conjugate & 106,936 & $69,489-174,511$ \\
PSII-CRM & 2 & - \\
\hline
\end{tabular}

Next, the functionality of the antibodies was verified by the in vitro toxin neutralization assay of pooled sera from mice immunized with the conjugates. Remarkably, as shown in Figure 4, both PSII conjugates elicited toxin neutralizing activity comparable to that induced by their respective protein carrier in unconjugated form, indicating that chemical conjugation did not alter the critical epitopes of recombinant toxin A and B fragments. As previously demonstrated [19], TcdA_B2 and TcdB_GT induce neutralizing antibodies only against the cognate toxin (data not shown), suggesting that vaccination should include combinations of fragments derived from each toxin to achieve the concurrent neutralization of TcdA and TcdB. 
Figure 4. Neutralization titers induced by PSII-conjugated toxin fragments. Sera were collected from mice immunized with TcdA_B2 and TcdB_GT fragments, either alone or conjugated to PSII, in the presence of MF59 adjuvant. Titers were defined as the reciprocal of the highest dilution able to inhibit 100\% rounding in IMR-90 human fibroblasts treated with $1 \mathrm{CTU}_{100}$ of toxin A (white column) or B (grey column). Pre-immune sera were used as negative controls. Values reported in the graph represent the mean dilution from three to five independent experiments. The horizontal bars indicate the Standard error.

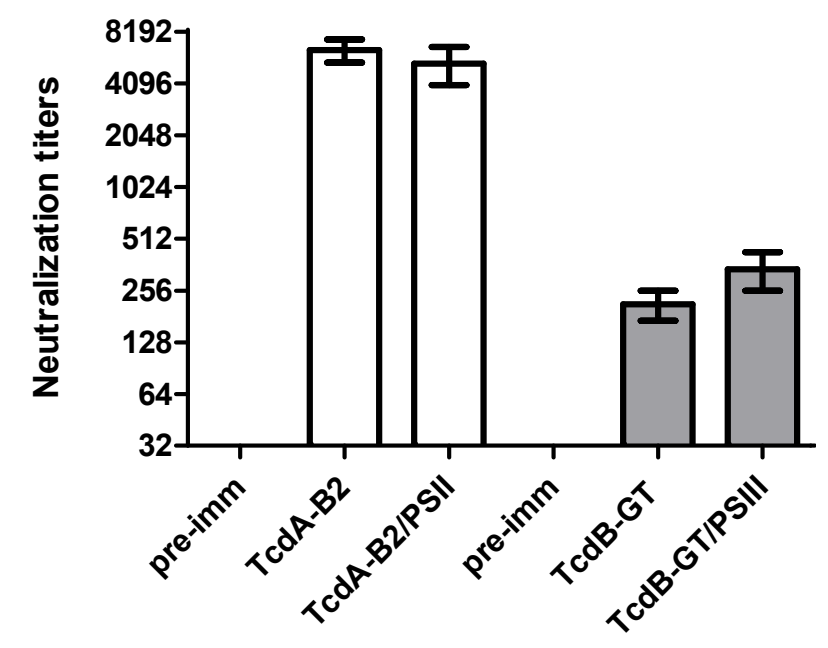

\section{Experimental Section}

\subsection{Preparation and Purification of PSII Glycoconjugates}

PSII at avDP 15 was obtained as previously reported in literature. Purified PSII [23] was reduced at the mannose sugar with $50 \mathrm{mM} \mathrm{NaBH}_{4}$ (Sigma, St. Louis, MO, USA) in $10 \mathrm{mM}$ sodium phosphate with $\mathrm{pH}=9.0$ at room temperature for $2 \mathrm{~h}$; the reduced PSII was purified by Sephadex G25 chromatography (G\&E Healthcare, Uppsala, Sweden) in water and then oxidized with 15 equivalent of $\mathrm{NaIO}_{4}$ (Sigma) in $10 \mathrm{mM}$ sodium phosphate with $\mathrm{pH}=7.2$ at room temperature for $2 \mathrm{~h}$ at the dark. The oxidized PSII was then purified by Sephadex G25 chromatography in water. The oxidized PSII $(10 \mathrm{mg} / \mathrm{mL})$ was then conjugated to carrier proteins using a stoichiometry of 4:1 (weight PSII per weight protein) in $200 \mathrm{mM}$ sodium phosphate/1 $\mathrm{M} \mathrm{NaCl}$ buffer with $\mathrm{pH}=8.0$, and in presence of $\mathrm{NaBH}_{3} \mathrm{CN}\left(2: 1\right.$, weight PSII per weight $\left.\mathrm{NaBH}_{3} \mathrm{CN}\right)$. The mixture was incubated for $48-72 \mathrm{~h}$ at $37{ }^{\circ} \mathrm{C}$, mixing very gently with a magnetic stirrer. Conjugates were purified from excess of unconjugated PSII using size exclusion Superdex 75 chromatography (G\&E Healthcare) in $10 \mathrm{mM}$ sodium phosphate/10 $\mathrm{mM}$ $\mathrm{NaCl}$ buffer with $\mathrm{pH}=7.2$.

\subsection{Characterization of PSII Glycoconjugates}

Conjugates were characterized by SDS-PAGE using 7\% Tris-Acetate gels (NuPAGE, Invitrogen, Carlsbad, CA, USA). The samples (5 $\mu \mathrm{g}$ in term of protein) were added of $0.5 \mathrm{M}$ dithiothreitol $(1 / 5 \mathrm{v} / \mathrm{v})$ and NuPAGE LDS sample buffer $(1 / 5 \mathrm{v} / \mathrm{v})$. The mixtures were heated at $100{ }^{\circ} \mathrm{C}$ for $1 \mathrm{~min}$. The gel containing loaded samples was electrophoresed at $45 \mathrm{~mA}$ in NuPAGE Tris-Acetate SDS running buffer (20×, Invitrogen) and stained with Simply Blue Safe Stain (Invitrogen). 
Protein concentration was determined by Micro bicinchoninic acid (BCA) protein assay kit (Thermo Scientific, Rockford, IL, USA). Total saccharide concentration was determined by high performance anionic exchange chromatography-pulsed amperometric detection (HPAEC-PAD) analysis. Unconjugated saccharide was separated by SPE C4 hydrophobic interaction column $(0.5 \mathrm{~mL}$ resin, Bioselect, Grace Vydac, Columbia, MD, USA) and subsequently estimated by HPAEC-PAD analysis.

\subsection{Cloning, Expression and Purification of Recombinant Fragments}

TcdA_B2 and TcdB_GT were cloned, expressed and purified as previously described [19]. Briefly, fragments were cloned in pet15b+ vector (N-term-His tag) using the Polymerase Incomplete Primer Extension (PIPE) method. The sequences coding for TcdA_B2 (residues 2303-2710 in TcdA) and TcdB_GT (residues 1-543 in TcdB) were amplified by PCR from the C. difficile strain 630 genomic DNA and the vector was amplified from the pet15b+ vector; E.coli HK100 cells were then transformed with vector/insert hybrids.

PIPE method was employed to generate TcdB_GT (D270A, R273A, Y284A, D286A and D288A) mutant with abrogated enzymatic activity.

The proteins were expressed in E.coli BL21 (DE3) cells (Novagen, Darmstadt, Germany) grown in LB. The expression of the protein was induced by addition of $1 \mathrm{mM}$ IPTG to the culture at exponential growth phase and incubation for $4 \mathrm{~h}$ at $25^{\circ} \mathrm{C}$.

The protein expression was checked by SDS-PAGE [19].

Recombinant TcdA_B2 and TcdB_GT were purified by immobilized metal ion affinity chromatography (IMAC) and buffer exchange was performed by PD-10 desalting column (G\&E Healthcare) or by dialysis. Protein quantification was performed by Micro BCA Protein Assay Kit (Thermo Scientific).

\subsection{Immunization Protocol}

Animal experimental guidelines set forth by the Novartis Animal Care Department were followed in the conduct of all animal studies.

Groups of eight female BALB/c mice (5-6 weeks old) were immunized on days 1, 21 and 35 with $2.5 \mu \mathrm{g}$ of conjugated carbohydrate antigen formulated with MF59 (mixing equal volume of conjugate and MF59 suspension) as adjuvants. All immunizations were performed by administering a $200 \mu \mathrm{L}$ of vaccine via intraperitoneal route. Adjuvant alone was used for negative control groups. Sera were collected on days 0 (before the first immunization), 34 and 49 (two weeks after the third immunization).

\subsection{ELISA Assay}

Specific antibodies titers were determined two weeks after the third immunization by ELISA assay. For that purpose 96-well Maxisorp plates (Nunc, Thermo Fisher Scientific, Roskilde, Denmark) were coated with $100 \mu \mathrm{L} /$ well of $2 \mu \mathrm{g} / \mathrm{mL}$ (protein content) PSII-HSA conjugate (prepared as previously reported [23]) or TcdA_B2 protein or TcdB_GT protein in PBS with $\mathrm{pH}=7.2$. Plates were incubated over night at $4{ }^{\circ} \mathrm{C}$, then washed three times with TPBS $(0.05 \%$ Tween 20 in PBS, pH $=7.4)$ and blocked with $100 \mu \mathrm{L} /$ well of $3 \%$ BSA (Sigma-Aldrich, St. Louis, MO, USA) for $1 \mathrm{~h}$ at $37{ }^{\circ} \mathrm{C}$. Each incubation 
step was always followed by triple TPBS wash. Serum samples were initially diluted 1:1000 in TPBS, transferred into coated-blocked plates $(200 \mu \mathrm{L})$ and serially two-fold diluted followed by $2 \mathrm{~h}$ incubation at $37^{\circ} \mathrm{C}$. Then $100 \mu \mathrm{L} /$ well of 1:10,000 diluted alkaline phosphatase-conjugated goat anti-mouse $\operatorname{IgG}$ (Sigma-Aldrich) were added and left for $1 \mathrm{~h}$ at $37^{\circ} \mathrm{C}$. Visualization of bound alkaline phosphatase was performed by adding $100 \mu \mathrm{L} /$ well of $1 \mathrm{mg} / \mathrm{mL}$ para-nitrophenyl-phosphate (pNPP) disodium hexahydrate (Sigma-Aldrich) in $0.5 \mathrm{M}$ diethanolamine buffer with $\mathrm{pH}=9.6$. After $30 \mathrm{~min}$ of development at room temperature, the optical density (OD) of each sample was measured at $405 \mathrm{~nm}$ with a microplate spectrophotometer (Biorad). Antibody titres were expressed as the reciprocal of sera dilution corresponding to a cut off $\mathrm{OD}=1.0$. Each group of immunization was represented as the geometrical mean (GMT) of the single mouse titers.

The statistical and graphical analysis was performed using GraphPad Prism 5 software (GraphPad Software Inc., La Jolla, CA, USA) by applying the Mann Whitney test for statistical analysis.

\subsection{In Vitro Neutralization Assay}

Toxin A and Toxin B were purified from C. difficile VPI10463 strain as previously described [19]. IMR-90 human fibroblasts were obtained from American Type Culture Collection (ATCC, Rockville, MD, USA). Cells were grown to $80 \%-90 \%$ confluence in 96 -well plates in Eagle's Minimum Essential Medium (EMEM, ATCC) with 10\% fetal calf serum. The minimal doses of Toxin A and Toxin B needed to cause $100 \%$ rounding in $24 \mathrm{~h}\left(1 \mathrm{CTU}_{100}\right)$ were defined as $20 \mathrm{ng} / \mathrm{mL}$ and $10 \mathrm{pg} / \mathrm{mL}$, respectively. For neutralization assay two-fold dilutions of mouse sera from 1:8 to 1:32,000 were pre-incubated with $1 \mathrm{CTU}_{100}$ of each toxin in cell medium for $90 \mathrm{~min}$ at $37{ }^{\circ} \mathrm{C}$. Sera and toxins mixtures were then added to the cells and incubated for 16-18 h before analysis. Pre-immune sera were used as negative controls. The endpoint titers were defined as the reciprocal of the highest dilution able to inhibit cell rounding.

\section{Conclusions}

C. difficile is considered the most important identifiable cause of healthcare-acquired diarrhea. The tendency of CDI to relapse and lack of efficacious preventative therapies render a vaccine highly recommendable. Typically, the two toxins A and B have been targeted for vaccine development. The surface polysaccharide PSII conjugated to $\mathrm{CRM}_{197}$ has also been proven an optimal target for a carbohydrate-based vaccine. In the present study, we explored the feasibility of a glycoconjugate vaccine where the PSII saccharide was conjugated to the two protein fragments TcdA_B2 and TcdB_GT from toxin A and B, respectively.

We demonstrated that TcdB_GT is a very efficient carrier for PSII, since PSII-TcdB_GT was highly immunogenic and induced high titers of anti-polysaccharide IgG antibodies, in a comparable manner to the PSII-CRM ${ }_{197}$ conjugate. On the other hand, the PSII-TcdA_B2 conjugate was less efficient in inducing anti-PSII IgGs. Since the chemical characteristics of the two conjugates are comparable, the reason for this different behavior might reside in the different intrinsic ability of the two carriers to drive the antibody response toward the carbohydrate moiety.

It is important to note that conjugation to the polysaccharide does not impact the neutralizing activity of both TcdA_B2 and TcdB_GT. Therefore, we conclude that conjugation of C. difficile 
carbohydrate antigens to toxin fragments is a promising approach for the design of a conjugate vaccine which targets both surface exposed carbohydrate as well as secreted toxins. Further evaluation in suitable animal models is needed to fully understand the capacity of such constructs to prevent colonization and neutralize toxin activity.

\section{Acknowledgments}

The authors thank Novartis Vaccines for the support to the project.

\section{Author Contributions}

Maria R. Romano, Rosanna Leuzzi, Francesco Berti, Paolo Costantino, Roberto Adamo and Maria Scarselli designed research; Maria R. Romano, Rosanna Leuzzi, Marta Tontini, Alberto Nilo, Daniela Proietti, Francesco Berti, Roberto Adamo, and Emilia Cappelletti performed research; Maria R. Romano, Rosanna Leuzzi, Francesco Berti, Paolo Costantino, Roberto Adamo and Maria Scarselli analyzed data; and Maria R. Romano, Rosanna Leuzzi and Roberto Adamo wrote the paper.

\section{Conflicts of Interest}

The authors declare no conflict of interest.

\section{References}

1. Kelly, C.P.; LaMont, J.T. Clostridium difficile-More difficult than ever. N. Engl. J. Med. 2008, 359, 1932-1940.

2. Rebeaud, F.; Bachmann, M.F. Immunization strategies for Clostridium difficile infections. Expert Rev. Vaccines 2012, 11, 469-479.

3. Chitnis, A.S.; Holzbauer, S.M.; Belflower, R.M.; Winston, L.G.; Bamberg, W.M.; Lyons, C.; Farley, M.M.; Dumyati, G.K.; Wilson, L.E.; Beldavs, Z.G.; et al. Epidemiology of community-associated Clostridium difficile infection, 2009 through 2011. JAMA Intern. Med. 2013, 173, 1359-1367.

4. Kyne, L.; Hamel, M.B.; Polavaram, R.; Kelly, C.P. Health care costs and mortality associated with nosocomial diarrhea due to Clostridium difficile. Clin. Infect. Dis. 2002, 34, 346-353.

5. Petrella, L.A.; Sambol, S.P.; Cheknis, A.; Nagaro, K.; Kean, Y.; Sears, P.S.; Babakhani, F.; Johnson, S.; Gerding, D.N. Decreased cure and increased recurrence rates for Clostridium difficile infection caused by the epidemic C. difficile BI strain. Clin. Infect. Dis. 2012, 55, 351-357.

6. Kyne, L.; Warny, M.; Qamar, A.; Kelly, C.P. Association between antibody response to toxin A and protection against recurrent Clostridium difficile diarrhoea. Lancet 2001, 357, 189-193.

7. Leav, B.A.; Blair, B.; Leney, M.; Knauber, M.; Reilly, C.; Lowy, I.; Gerding, D.N.; Kelly, C.P.; Katchar, K.; Baxter, R.; et al. Serum anti-toxin B antibody correlates with protection from recurrent Clostridium difficile infection (CDI). Vaccine 2010, 28, 965-969. 
8. Steele, J.; Mukherjee, J.; Parry, N.; Tzipori, S. Antibody against TcdB, but not TcdA, prevents development of gastrointestinal and systemic Clostridium difficile disease. J. Infect. Dis. 2013, 207, 323-330.

9. Jank, T.; Giesemann, T.; Aktories, K. Rho-glucosylating Clostridium difficile toxins A and B: New insights into structure and function. Glycobiology 2007, 17, 15R-22R.

10. Lowy, I.; Molrine, D.C.; Leav, B.A.; Blair, B.M.; Baxter, R.; Gerding, D.N.; Nichol, G.; Thomas, W.D., Jr.; Leney, M.; Sloan, S.; et al. Treatment with monoclonal antibodies against Clostridium difficile toxins. N. Engl. J. Med. 2010, 362, 197-205.

11. Marozsan, A.J.; Ma, D.; Nagashima, K.A.; Kennedy, B.J.; Kang, Y.K.; Arrigale, R.R.; Donovan, G.P.; Magargal, W.W.; Maddon, P.J.; Olson, W.C. Protection against Clostridium difficile infection with broadly neutralizing antitoxin monoclonal antibodies. J. Infect. Dis. 2012, 206, 706-713.

12. Kotloff, K.L.; Wasserman, S.S.; Losonsky, G.A.; Thomas, W., Jr.; Nichols, R.; Edelman, R.; Bridwell, M.; Monath, T.P. Safety and immunogenicity of increasing doses of a Clostridium difficile toxoid vaccine administered to healthy adults. Infect. Immun. 2001, 69, 988-995.

13. Aboudola, S.; Kotloff, K.L.; Kyne, L.; Warny, M.; Kelly, E.C.; Sougioultzis, S.; Giannasca, P.J.; Monath, T.P.; Kelly, C.P. Clostridium difficile vaccine and serum immunoglobulin G antibody response to toxin A. Infect. Immun. 2003, 71, 1608-1610.

14. Sougioultzis, S.; Kyne, L.; Drudy, D.; Keates, S.; Maroo, S.; Pothoulakis, C.; Giannasca, P.J.; Lee, C.K.; Warny, M.; Monath, T.P.; et al. Clostridium difficile toxoid vaccine in recurrent C. difficile-associated diarrhea. Gastroenterology 2005, 128, 764-770.

15. Donald, R.G.; Flint, M.; Kalyan, N.; Johnson, E.; Witko, S.E.; Kotash, C.; Zhao, P.; Megati, S.; Yurgelonis, I.; Lee, P.K.; et al. A novel approach to generate a recombinant toxoid vaccine against Clostridium difficile. Microbiology 2013, 159, 1254-1266.

16. Ryan, E.T.; Butterton, J.R.; Smith, R.N.; Carroll, P.A.; Crean, T.I.; Calderwood, S.B. Protective immunity against Clostridium difficile toxin A induced by oral immunization with a live, attenuated Vibrio cholerae vector strain. Infect. Immun. 1997, 65, 2941-2949.

17. Ward, S.J.; Douce, G.; Figueiredo, D.; Dougan, G.; Wren, B.W. Immunogenicity of a Salmonella typhimurium aroA aroD vaccine expressing a nontoxic domain of Clostridium difficile toxin A. Infect. Immun. 1999, 67, 2145-2152.

18. Tian, J.H.; Fuhrmann, S.R.; Kluepfel-Stahl, S.; Carman, R.J.; Ellingsworth, L.; Flyer, D.C. A novel fusion protein containing the receptor binding domains of $C$. difficile toxin $\mathrm{A}$ and toxin $\mathrm{B}$ elicits protective immunity against lethal toxin and spore challenge in preclinical efficacy models. Vaccine 2012, 30, 4249-4258.

19. Leuzzi, R.; Spencer, J.; Buckley, A.; Brettoni, C.; Martinelli, M.; Tulli, L.; Marchi, S.; Luzzi, E.; Irvine, J.; Candlish, D.; et al. Protective efficacy induced by recombinant Clostridium difficile toxin fragments. Infect. Immun. 2013, 81, 2851-2860.

20. Spencer, J.; Leuzzi, R.; Buckley, A.; Irvine, J.; Candlish, D.; Scarselli, M.; Douce, G.R. Vaccination against Clostridium difficile using toxin fragments: Observations and analysis in animal models. Gut Microbes 2014, 5, doi:10.4161/gmic.27712. 
21. Monteiro, M.A.; Ma, Z.; Bertolo, L.; Jiao, Y.; Arroyo, L.; Hodgins, D.; Mallozzi, M.; Vedantam, G.; Sagermann, M.; Sundsmo, J.; et al. Carbohydrate-based Clostridium difficile vaccines. Expert Rev. Vaccines 2013, 12, 421-431.

22. Ganeshapillai, J.; Vinogradov, E.; Rousseau, J.; Weese, J.S.; Monteiro, M.A. Clostridium difficile cell-surface polysaccharides composed of pentaglycosyl and hexaglycosyl phosphate repeating units. Carbohydr. Res. 2008, 343, 703-710.

23. Adamo, R.; Romano, M.R.; Berti, F.; Leuzzi, R.; Tontini, M.; Danieli, E.; Cappelletti, E.; Cakici, O.S.; Swennen, E.; Pinto, V.; et al. Phosphorylation of the synthetic hexasaccharide repeating unit is essential for the induction of antibodies to Clostridium difficile PSII cell wall polysaccharide. ACS Chem. Biol. 2012, 7, 1420-1428.

24. Reid, C.W.; Vinogradov, E.; Li, J.; Jarrell, H.C.; Logan, S.M.; Brisson, J.R. Structural characterization of surface glycans from Clostridium difficile. Carbohydr. Res. 2012, 354, 65-73.

25. Đapa, T.; Leuzzi, R.; Ng, Y.K.; Baban, S.T.; Adamo, R.; Kuehne, S.A.; Scarselli, M.; Minton, N.P.; Serruto, D.; Unnikrishnan, M. Multiple factors modulate biofilm formation by the anaerobic pathogen Clostridium difficile. J. Bacteriol. 2013, 195, 545-555.

26. Berti, F.; Adamo, R. Recent mechanistic insights on glycoconjugate vaccines and future perspectives. ACS Chem. Biol. 2013, 8, 1653-1663.

27. Bertolo, L.; Boncheff, A.G.; Ma, Z.; Chen, Y.H.; Wakeford, T.; Friendship, R.M.; Rosseau, J.; Weese, J.S.; Chu, M.; Mallozzi, M.; et al. Clostridium difficile carbohydrates: Glucan in spores, PSII common antigen in cells, immunogenicity of PSII in swine and synthesis of a dual C. difficile-ETEC conjugate vaccine. Carbohydr. Res. 2012, 354, 79-86.

28. Bröker, M.; Costantino, P.; DeTora, L.; McIntosh, E.D.; Rappuoli, R. Biochemical and biological characteristics of cross-reacting material 197 CRM197, a non-toxic mutant of diphtheria toxin: Use as a conjugation protein in vaccines and other potential clinical applications. Biologicals 2011, 39, 195-204.

29. Costantino, P.; Rappuoli, R.; Berti, F. The design of semi-synthetic and synthetic glycoconjugate vaccines. Expert Opin. Drug Discov. 2011, 6, 1045-1067.

30. Oberli, M.A.; Hecht, M.L.; Bindschädler, P.; Adibekian, A.; Adam, T.; Seeberger, P.H. A possible oligosaccharide-conjugate vaccine candidate for Clostridium difficile is antigenic and immunogenic. Chem. Biol. 2011, 18, 580-588.

31. Danieli, E.; Lay, L.; Proietti, D.; Berti, F.; Costantino, P.; Adamo, R. First synthesis of C. difficile PS-II cell wall polysaccharide repeating unit. Org. Lett. 2011, 13, 378-381.

32. Galli, G.; Medini, D.; Borgogni, E.; Zedda, L.; Bardelli, M.; Malzone, C.; Nuti, S.; Tavarini, S.; Sammicheli, C.; Hilbert, A.K.; et al. Adjuvanted H5N1 vaccine induces early $\mathrm{CD}^{+} \mathrm{T}$ cell response that predicts long-term persistence of protective antibody levels. Proc. Natl. Acad. Sci. USA 2009, 106, 3877-3882.

(C) 2014 by the authors; licensee MDPI, Basel, Switzerland. This article is an open access article distributed under the terms and conditions of the Creative Commons Attribution license (http://creativecommons.org/licenses/by/3.0/). 